\title{
Macroscopic Quantum Tunneling of a Domain Wall in a Ferromagnetic Metal
}

\author{
Gen Tatara and Hidetoshi Fukuyama \\ Department of Physics, University of Tokyo, 7-3-1 Hongo, Tokyo 113, Japan
}

(October 8, 2018)

\begin{abstract}
The macroscopic quantum tunneling of a planar domain wall in a ferromagnetic metal is studied based on the Hubbard model. It is found that the ohmic dissipation is present even at zero temperature due to the gapless Stoner excitation, which is the crucial difference from the case of the insulating magnet. The dissipative effect is calculated as a function of width of the wall and is shown to be effective in a thin wall and in a weak ferromagnet. The results are discussed in the light of recent experiments on ferromagnets with strong anisotropy.
\end{abstract}

Typeset using REVTEX 
In recent years, owing mainly to the development of technology in mesoscopic physics, there has been growing interest in macroscopic quantum tunneling (MQT) in magnetic systems [1], e.g., the magnetization reversal in small grains [2], the quantum nucleation of a domain [3], and the quantum depinning of a domain wall via MQT [4]. These studies are mainly in ferromagnets, but recently a magnetization reversal due to MQT has been observed in antiferromagnetic particles of horse spleen ferritin [5]. In the case of the quantum depinning of a domain wall pinned by defects, the position of the wall at the pinning center becomes metastable in the external magnetic field, and if the barrier height is low enough, the position tunnels out of the local minimum. This problem was studied theoretically by Stamp [4] for the case of an insulating magnet. The tunneling rate was expressed in terms of macroscopic variables, and was shown to be large enough to be observed even for a large wall with about $10^{10}$ spins. As sources of dissipation, which is shown to be important by the seminal paper by Caldeira and Leggett [6], Stamp considered magnons and phonons, but the effects turn out to be negligible, since magnon has a gap and coupling to phonon is weak. Consequently it has been concluded that the tunneling rate is not essentially affected by dissipation in insulators.

Experiments on MQT in magnetic system, however, have been carried out in metallic ferromagnets. In metals, in contrast to the case of insulators, there is a gapless excitation of spin flip, and hence dissipation from conduction electrons must be very important. Consequently the quantum motion of the wall in metals should be quite different from that in insulators. [7] In this paper, we will investigate theoretically the dissipative effect on MQT of a domain wall in a ferromagnetic metal based on an itinerant electron model. An important and interesting feature of the itinerant system is that the electron, which supports magnetization, works also as a source of dissipation in the dynamical motion of the magnetization itself. Our analysis is based on the Hubbard model in the continuum. The calculation is carried out at zero temperature, since we are interested only in the quantum tunneling present at low temperature.

The Lagrangian in the imagnary time path integral is given by 


$$
L=\sum_{\mathbf{k} \sigma} c_{\mathbf{k} \sigma}^{\dagger}\left(\partial_{\tau}+\epsilon_{\mathbf{k}}\right) c_{\mathbf{k} \sigma}+U \sum_{\mathbf{x}} n_{\mathbf{x} \uparrow} n_{\mathbf{x} \downarrow}
$$

where $c_{\mathbf{x} \sigma}$ is an electron operator at site $\mathbf{x}$ with spin $\sigma(= \pm), n_{\mathbf{x} \sigma} \equiv c_{\mathbf{x} \sigma}^{\dagger} c_{\mathbf{x} \sigma}$ and $U$ is the Coulomb repulsion. The band energy is $\epsilon_{\mathbf{k}} \equiv \mathbf{k}^{2} /(2 m)-\epsilon_{\mathrm{F}}$ with the fermi energy $\epsilon_{\mathrm{F}}$. The Coulomb repulsion term will be rewritten by introducing a Hubbard-Stratonovich field representing the magnetization; $\mathbf{M}_{\mathbf{x}} \equiv M_{\mathbf{x}} \mathbf{n}_{\mathbf{x}}$, where $M_{\mathbf{x}} \equiv<\left(c^{\dagger} \boldsymbol{\sigma} c\right)_{\mathbf{x}}>\mathbf{n}_{\mathbf{x}}$ with $\mathbf{n}_{\mathbf{x}}$ being a slowly varying unit vector which describes the direction of magnetization. The magnitude of magnetization is assumed as space-time independent, $M_{\mathbf{x}} \equiv M$. Hence only $\mathbf{n}_{\mathbf{x}}$ remains as the relevant degree of freedom.

The spatial variation of $\mathbf{n}_{\mathbf{x}}$ accompanied with a domain wall is assumed to be much slower compared to the inverse fermi momentum of the electron $k_{\mathrm{F}}^{-1}$. For the analysis of such a slowly varying field, a locally rotated frame [8] of electron is convenient such that the $z$-axis of the electron is chosen in the direction of the local magnetization vector $\mathbf{n}_{\mathbf{x}}$. The electron operator $a_{\mathbf{x} \sigma}$ in the new frame is related to the original $c_{\mathbf{x} \sigma}$ as

$$
a_{\mathbf{x} \sigma} \equiv \sigma \cos (\theta / 2) c_{\mathbf{x} \sigma}+e^{-i \sigma \phi} \sin (\theta / 2) c_{\mathbf{x},-\sigma}
$$

where the polar coordinates $\left(\theta_{\mathbf{x}}(\tau), \phi_{\mathbf{x}}(\tau)\right)$ parametrize the direction of $\mathbf{n}_{\mathbf{x}}(\tau)$. The electron $a_{\mathbf{x} \sigma}$ is polarized uniformly with the energy $\epsilon_{\mathbf{k} \sigma} \equiv \mathbf{k}^{2} / 2 m-\sigma U M-\epsilon_{\mathrm{F}}$. As a price of this transformation, there arises from the kinetic term $c^{\dagger} \dot{c}+|\nabla c|^{2} /(2 m)$ an additional term $H_{\text {int }}$ that describes the interaction of electrons with spatial variation of the magnetization vector [8]. This interaction $H_{\text {int }}$ is small and of the order of $O\left(k_{\mathrm{F} \uparrow} \lambda\right)^{-1}$, where $\lambda$ is the domain wall thickness, $k_{\mathrm{F} \uparrow}$ is the fermi momentum of the majority spin, and hence can be treated perturbatively. Our following results are valid for $\lambda k_{\mathrm{F} \uparrow} \gtrsim 1$.

The integration over the electron degrees of freedom leads to the effective action for the magnetization as $S_{\text {eff }}=-\operatorname{tr} \ln \left(\partial_{\tau}+\epsilon_{\mathbf{k} \sigma}\right)+\beta \sum_{\mathbf{x}}(U / 2) M^{2}+\Delta S$. The first two terms are the mean field action for a ferromagnet which determines the magnetization $M$. The dynamics of $(\theta, \phi)$ is described by $\Delta S$, which is expressed in terms of correlation functions of electron. This term is decomposed into two parts, that is local and non-local in time, respectively, as $\Delta S \equiv \Delta S_{\mathrm{loc}}+\Delta S_{\mathrm{dis}}$. The local part $\Delta S_{\mathrm{loc}}$ determines the dynamics of magnetization vector, 
and the non-local part $\Delta S_{\text {dis }}$ represents the dissipative effect due to conduction electrons on the motion of the magnetization vector.

Up to the lowest order in $\partial_{\tau}$ and $\nabla$, the local part $\Delta S_{\text {loc }}$ turns out to be formally the same as the ferromagnetic Heisenberg model [8,9] with spin $S \equiv M / 2$ whose Lagrangian is given by

$$
L_{\mathrm{H}}=\int d^{3} \mathbf{x}\left[i \frac{S}{a^{3}} \dot{\phi}(1-\cos \theta)+\frac{J S^{2}}{2}\left((\nabla \theta)^{2}+\sin ^{2} \theta(\nabla \phi)^{2}\right)\right] .
$$

The exchange coupling or the spin wave stiffness constant is expressed by the parameters of the original Hubbard model as $J \equiv\left(n / m a^{3} M^{2}\right)\left[1-\left(\left(k_{\mathrm{F} \uparrow}^{5}-k_{\mathrm{F} \downarrow}^{5}\right) a^{3}\right) /\left(30 \pi^{2} m n U M\right)\right]$, where $n$ is the electron number per site, $k_{\mathrm{F} \sigma} \equiv\left(2 m\left(\epsilon_{\mathrm{F}}+\sigma U M\right)\right)^{1 / 2}$ is the fermi momentum and $a$ is the lattice constant. Hence, in the absence of the non-local term $\Delta S_{\text {dis }}$, there is no formal difference between metallic and insulating ferromagnets, and the tunneling rate of the domain wall is determined on the same footing [4].

In order to incorperate the domain wall, the anisotropy energy with $y z$ easy plane is introduced [10];

$$
H_{\mathrm{ani}}=\int d^{3} \mathbf{x}\left(-\frac{K}{2} S^{2} \cos ^{2} \theta+\frac{K_{\perp}}{2} S^{2} \sin ^{2} \theta \cos ^{2} \phi\right)
$$

The Lagrangian $L_{\mathrm{H}}+H_{\text {ani }}$ has a planar domain wall centered at $x=Q(\tau)$ and moving slowly as a classical solution; $\cos \theta(\mathbf{x}, \tau)=\tanh (x-Q(\tau) / \lambda)$ and $\cos \phi(\mathbf{x}, \tau) \simeq i \dot{Q} / c \ll 1$ with $c \equiv K_{\perp} \lambda S a^{3}$ where $\lambda \equiv \sqrt{J / K}$ is the width of the wall. This configuration is depicted in Fig. 11.

For the magnetic field $H$ close to the coercive field $H_{c}$, i.e., $\left(H_{c}-H\right) / H_{c} \equiv \epsilon \ll 1$, the potential for the wall coordinate $Q$ is given by $V(Q) \equiv(1 / 2) M_{w} \omega_{0}^{2} Q^{2}\left[1-\left(Q / Q_{0}\right)^{2}\right]$ where $M_{w} \equiv 2 N /\left(K_{\perp} \lambda^{2} a^{3}\right)$ is the domain wall mass $N$ being the number of the spins in the wall. For this case of small $\epsilon$, the attempt frequency around the minimum is $\omega_{0} \simeq\left(\mu_{0}(\hbar \gamma)^{2} / a^{3}\right) \sqrt{h_{c}} \epsilon^{\frac{1}{4}}$ and the width of the barrier is given by $Q_{0}=\sqrt{3 / 2} \sqrt{\epsilon} \lambda$ where $h_{c} \equiv H_{c} /\left(\hbar \gamma S / a^{3}\right)$ is the ratio of the coercive field to the magnetic moment per unit volume $\left(\mu_{0}\right.$ is the magnetic peameability of free space and $\gamma$ is the gyromagnetic ratio). The actual value of attempt 
frequency is $\omega_{0} \simeq 5 \times \sqrt{h_{c}} \epsilon^{\frac{1}{4}}(\mathrm{~K})$ for the choice of $a=3 \AA$, and in the present case, this is roughly the same as the crossover temperature $T_{c o}$ from the thermal activation to the quantum tunneling. The classical solution (bounce) of $Q$ in the metastable potential $V(Q)$ is given by $Q(\tau)=Q_{0} / \cosh ^{2}\left(\omega_{0} \tau / 2\right)$, and the tunneling rate out of the local minimum is estimated by use of this bounce solution. For the case of the wall with the cross sectional area $N a^{3} / \lambda$ as shown in Fig. 1, the rate $\Gamma_{0}$ without dissipation is reduced to $\Gamma_{0}=A \exp (-B)$ where $A \simeq\left(\mu_{0}(\hbar \gamma)^{2} / a^{3}\right) N^{1 / 2} h_{c}^{3 / 4} \epsilon^{7 / 8} \simeq 10^{11} N^{1 / 2} h_{c}^{3 / 4} \epsilon^{7 / 8}(\mathrm{~Hz})$ and the exponent, $B$, is given as $B \simeq N h_{c}^{1 / 2} \epsilon^{5 / 4}$. [4] Since $B$ is proportional to $N \epsilon^{5 / 4}$ [1], a small value of $\epsilon$ is needed to observe the tunneling.

Let us now look into the non-local action $\Delta S_{\text {dis }}$, where the characteristic feature of the itinerant electron system is to be seen. For the case of a weak dissipation, this contribution is evaluated by use of the configuration of a domain wall obtained in the absence of dissipation. Up to $\nabla^{2}, \Delta S_{\text {dis }}$ is obtained as

$$
\begin{aligned}
& \Delta S_{\text {dis }}=\frac{1}{(4 m)^{2}} \int d \tau \int d \tau^{\prime} \frac{1}{\beta} \sum_{\ell} e^{i \omega_{\ell}\left(\tau-\tau^{\prime}\right)} \sum_{i} \sum_{\mathbf{q}} q_{i}^{2} \\
& \times\left|\theta_{\mathbf{q}}(\tau)-\theta_{\mathbf{q}}\left(\tau^{\prime}\right)\right|^{2}<J_{+}^{i}(\mathbf{q}) J_{-}^{i}(-\mathbf{q})>\left.\right|_{i \omega_{\ell}},
\end{aligned}
$$

where $\mathbf{J}_{\alpha}(\mathbf{q})(\alpha= \pm, z)$ are the Fourier transform of the spin currents of the electron; $\mathbf{J}_{\alpha} \equiv-i\left[\left(a^{\dagger} \sigma_{\alpha} \nabla a\right)-\left(\nabla a^{\dagger} \sigma_{\alpha} a\right)\right]$ with $\sigma_{ \pm} \equiv \sigma_{x} \pm i \sigma_{y}$, and $\theta_{\mathbf{q}} \equiv \sum_{\mathbf{x}} e^{-i \mathbf{q x}} \theta_{\mathbf{x}}$. The dissipation does not result from the $z$-component $J_{z}$ in the present case of a domain wall motion with $\nabla \phi=0$. The expectation value of electron spin current $\left\langle J_{+} J_{-}>\right.$in $\Delta S_{\text {dis }}$ is evaluated by the random phase approximation (RPA) in the background of uniform magnetization. [12]

After the analytic continuation to real frequency, $\Delta S_{\text {dis }}$ is expressed by the imaginary part of the retarded correlation function $\left.\left\langle J_{+} J_{-}\right\rangle\right|_{\omega+i 0}$ as 13

$$
\begin{aligned}
& \Delta S_{\text {dis }}=\frac{1}{(4 m)^{2}} \int d \tau \int d \tau^{\prime} \sum_{\mathbf{q}} q_{x}^{2}\left|\theta_{\mathbf{q}}(\tau)-\theta_{\mathbf{q}}\left(\tau^{\prime}\right)\right|^{2} \\
& \times \int_{0}^{\infty} \frac{d \omega}{\pi} e^{-\omega\left|\tau-\tau^{\prime}\right|} \operatorname{Im}<J_{+}^{x}(\mathbf{q}) J_{-}^{x}(-\mathbf{q})>\left.\right|_{\omega+i 0} .
\end{aligned}
$$

The imaginary part is expanded in terms of $\omega / \epsilon_{\mathrm{F}}$ as 
$\operatorname{Im}<\mathbf{J}_{+}^{x}(\mathbf{q}) \mathbf{J}_{-}^{x}(-\mathbf{q})>\left.\right|_{\omega+i 0}=\left\{\begin{array}{cc}\omega \frac{m^{2} a^{3}\left(k_{\mathrm{F} \uparrow}^{2}-k_{\mathrm{F} \downarrow}^{2}\right)^{2}}{\pi|q|^{3}}+O\left(\omega^{3}\right) & k_{\mathrm{F} \uparrow}-k_{\mathrm{F} \downarrow}<|q|<k_{\mathrm{F} \uparrow}+k_{\mathrm{F} \downarrow} \\ 0 & \text { otherwise. }\end{array}\right.$

The term linear in $\omega$ gives rise to the ohmic dissipation. It is seen from the restriction on $q$ that the ohmic dissipation arises from the Stoner excitation, which is a gapless excitation of spin flip across the fermi energy.

By the expression of the domain wall configuration, the non-local part of the effective action is reduced to

$$
\begin{aligned}
& \Delta S_{\mathrm{dis}}=N \frac{\left(k_{\mathrm{F} \uparrow}^{2}-k_{\mathrm{F} \downarrow}^{2}\right)^{2} a^{4}}{4} \frac{1}{\lambda a} \int d \tau \int d \tau^{\prime} \frac{1}{\left(\tau-\tau^{\prime}\right)^{2}} \\
& \times \int_{k_{\mathrm{F} \uparrow}-k_{\mathrm{F} \downarrow}}^{k_{\mathrm{F} \uparrow}+k_{\mathrm{F} \downarrow}} \frac{d q}{2 \pi} \sin ^{2} \frac{q}{2}\left(Q(\tau)-Q\left(\tau^{\prime}\right)\right) \frac{1}{q^{3}} \frac{1}{\cosh ^{2} \frac{\pi}{2} \lambda q} .
\end{aligned}
$$

The form factor of the wall, $1 / \cosh ^{2}(\pi \lambda q / 2)$, represents the effective coupling between electrons and the wall, and because of this factor, the momentum integration is dominated by $q \lesssim \lambda^{-1}$. The time integral is estimated by approximating the bounce solution as $Q(\tau) \simeq Q_{0} \theta\left(\omega_{0}^{-1}-|\tau|\right)$ and by introducing a short time cutoff of $\omega_{0}^{-1}$ for the relative time $\left(\tau-\tau^{\prime}\right)$ [14. Noting $q Q_{0} \propto q \lambda \sqrt{\epsilon} \ll 1$, the sine function in Eq. (\$) can be replaced by its argument and the action is evaluated to be $\Delta S_{\text {dis }} \equiv \eta N \epsilon$ where the factor, $\epsilon$, is due to the smallness of the squared tunnel distance $Q_{0}^{2}$. Here the strength of dissipation, $\eta$, is

$$
\eta=\frac{3 \ln 3}{16 \pi}\left(k_{\mathrm{F} \uparrow}^{2}-k_{\mathrm{F} \downarrow}^{2}\right)^{2} a^{4} \frac{\lambda}{a} \int_{\left(k_{\mathrm{F} \uparrow}-k_{\mathrm{F} \downarrow}\right) \frac{\pi}{2} \lambda}^{\left(k_{\mathrm{F} \uparrow}+k_{\mathrm{F} \downarrow}\right) \frac{\pi}{2} \lambda} d x \frac{1}{x} \frac{1}{\cosh ^{2} x} .
$$

For a thick wall $\lambda\left(k_{\mathrm{F} \uparrow}-k_{\mathrm{F} \downarrow}\right) \gg 1, \eta \propto \exp \left[-\pi \lambda\left(k_{\mathrm{F} \uparrow}-k_{\mathrm{F} \downarrow}\right)\right]$ and then the dissipation is negligible. On the other hand, $\eta$ can be large if $\left(k_{\mathrm{F} \uparrow}-k_{\mathrm{F} \downarrow}\right) \lambda \lesssim 1$. This condition is compatible with that of slow spatial variation $\lambda k_{\mathrm{F} \uparrow} \gtrsim 1$ for a wall with moderate thickness in a weak ferromagnet and for a thin wall in a stronger ferromagnet. The strength $\eta$ is plotted as a function of $(\lambda / a)$ in Fig. 2 for three different values of $\delta \equiv\left(k_{\mathrm{F} \uparrow}-k_{\mathrm{F} \downarrow}\right) /\left(k_{\mathrm{F} \uparrow}+k_{\mathrm{F} \downarrow}\right)$ with $\left(k_{\mathrm{F} \uparrow}+k_{\mathrm{F} \downarrow}\right) a=6$ which may represent the case of an iron. The dissipation is larger for weaker magnet (smaller $\delta$ ). (For a complete ferromagnet, $k_{\mathrm{F} \downarrow}$ vanishes and the ohmic dissipation disappears.) It is seen that $\eta$ can be of the order 0.1 for a wall with thickness 
a few times the lattice spacing with $\delta \lesssim 0.1$. In the presence of dissipation, the tunneling rate is reduced to be $\Gamma=A \exp \left[-\left(B+\Delta S_{\text {dis }}\right)\right]=\Gamma_{0} \exp (-\eta N \epsilon)$. Because of the different $\epsilon$-dependence of $B$ and $\Delta S_{\text {dis }}$, the ratio $\Delta S_{\text {dis }} / B=\eta h_{c}^{-1 / 2} \epsilon^{-1 / 4}$ is much larger than unity for the case of small $\epsilon$ we are interested in, and in particular for a thin wall ( $h_{c}$ is usually small, e.g., $\simeq 10^{-4}$ ). Consequently the tunneling rate is predominantly determined by dissipation in such cases. The tunneling rate $\Gamma$ is shown in Fig. 3 for the case of insulator $(\eta=0)$ and the typical case of a metal $(\eta=0.1)$ by the broken and solid lines, respectively for a choice of $h_{c}=10^{-4}$. In this figure, the number of spins is taken either $N=10^{4}$ or $10^{8}$. The value $N=10^{4}$ corresponds, for instance, to a wall with thickness of about $10 \AA$ and the area of $200 \AA \times 200 \AA$. The tunneling rate is seen to be much smaller in metals than that in insulators.

We have neglected the effect of magnetic field on electronic states. This is justified as long as $U M \gg \gamma H$. In experimental situations with the magnetic field of $\lesssim 1 \mathrm{~T}$ and $U \simeq 10 \mathrm{eV}$, this condition reduces to $M \gtrsim 10^{-4}$ in unit of the Bohr magneton, which is easy to satisfy. However, in order to discuss the case of very small $M$, the fluctuation of the magnitude $M_{\mathbf{x}}$ around the mean field value must also be included.

The contributions of higher order in $H_{\text {int }}$ are smaller than that of the second order we have calculated; for the potential renormalization by the order of $\left(k_{\mathrm{F} \uparrow} \lambda\right)^{-2}$ and for the dissipative effect by $\left(k_{\mathrm{F} \uparrow} \lambda\right)^{-2}$ or $\epsilon$.

In Eq. (9) we have taken account of only the ohmic dissipation. The super-ohmic contributions, which are of higher orders of $\left(\omega / \epsilon_{\mathrm{F}}\right)$ in Eq. (『), are smaller than the ohmic one by a factor of $\left(\omega_{0} / \epsilon_{\mathrm{F}}\right)^{2} \ll 1$ and hence are negligible. On the other hand, a contribution from the magnon pole, which has not been taken into account in the correlation function $<J J>$, is calculated from

$$
\operatorname{Im}<\mathbf{J}_{+}^{x}(\mathbf{q}) \mathbf{J}_{-}^{x}(-\mathbf{q})>\left.\right|_{\omega+i 0} ^{(\text {pole })} \simeq \pi M^{3}(J m q)^{2} \delta\left(\omega-\omega_{q}\right)
$$

where $\omega_{q} \equiv \Delta_{0}+J M a^{3} q^{2} / 2$ is the magnon energy with the anisotropy gap $\Delta_{0}$. This pole leads to super-ohmic dissipation, whose strength, $\eta^{(\text {pole })}$, is evaluated as $\eta^{(\text {pole })}=$ 
$(28 / 5) M\left(\Delta_{0} / \omega_{0}\right) \exp \left(-\Delta_{0} / \omega_{0}\right)$. Since experiments are usually carried out in highly anisotropic materials with $\Delta_{0} / \omega_{0} \simeq 10$, this contribution is very small compared to the ohmic dissipation for the case of a thin wall.

The present metallic case, where the ohmic dissipation is present even at absolute zero, are in contrast with the insulating case. At finite temperatures, however, there are ohmic dissipations even in the latter case. Stamp calculated such ohmic dissipations from two- and three-magnon processes and found $\eta^{(\mathrm{mag})}=(3 / 2 \pi S)\left(1 / \beta \Delta_{0}\right) \exp \left(-\beta \Delta_{0}\right)$. These processes corresponds to higher order contribution of $H_{\text {int }}$ in our calculation. In contrast to the case of metals, the value of $\eta^{(\mathrm{mag})}$ vanishes at $T=0$ and is very small at $\beta \Delta_{0} \gg 1$, hence the ratio of $\Delta S_{\text {dis }} / B$ is negligibly small in insulators.

In metals, eddy currents may influence MQT. An electric field is induced by Faraday's law from the change of magnetizations accompanied with the motion of the wall. This field produces the electric current and thus leads to the Joule heat of $P=\left(\mu_{0} \hbar \gamma / a^{3}\right)^{2} \dot{Q}^{2} / \rho$ per unit volume where $\rho$ is the resistivity. By use of the specific heat $C$ and the system size of $L$, the temperature rise due to the eddy current is expressed as $\Delta T=P L /(C \dot{Q})$. For $\rho \simeq 10^{-7}[\Omega \mathrm{m}], C \simeq 10\left[\mathrm{~J} / \mathrm{Km}^{3}\right]$, and $\dot{Q} \simeq 1[\mathrm{~m} / \mathrm{s}]$ with $L=100 \AA$, it is estimated as $\Delta T \simeq 10 \mathrm{mK}$. This value is not negligible but effects associated with this temperature rise may be separated from the intrinsic effects in careful experiments.

Our result shows a distinct difference between MQT of thin walls in metallic and insulating magnets. Unfortunately the experiments carried out so far appears not yet be able to observe dissipation due to itinerant electrons. In the experiment of a domain wall motion in a small particle of $\mathrm{Tb}_{0.5} \mathrm{Ce}_{0.5} \mathrm{Fe}_{2}, \mathrm{MQT}$ was observed below $T_{c o} \simeq 0.6 \mathrm{~K}$ [15]. However, the width of the domain wall is about $30 \AA$ and according to our result, $\eta \propto \exp \left[-\pi \lambda\left(k_{\mathrm{F} \uparrow}-k_{\mathrm{F} \downarrow}\right)\right]$, the dissipation from electron is negligible for such a thick wall. This may be the reason why the result of the crossover temperature $T_{c o} \sim 0.6 \mathrm{~K}$ is roughly in agreement with the theory [4] without dissipation. On the other hand, the domain wall in $\mathrm{SmCo}_{5}$ is very thin $\lambda \simeq 12 \AA$, and our result suggests strong effect of dissipation, which will be interesting to observe. Experiments on bulk crystal of $\mathrm{SmCo}_{3.5} \mathrm{Cu}_{1.5}$ with very thin walls (a few times $a$ ) have been 
performed [16], although quantitative argument is not easy since many walls participate in these experiments. Even in the case of thick walls, the dissipative effect becomes large in weak ferromagnets, where the experiments, however, will not be easy because of small value of saturation magnetization $M$.

MQT in disordered magnets has a new possiblity of observing a significant effect of sub-ohmic dissipation. In fact, as disorder is increased in a metallic magnet, the Anderson transition into an insulator will occur, and it was shown recently that near the transition the dissipation due to the conduction electron becomes sub-ohmic [13]. Disordered magnets may also be suitable for study of MQT because the eddy current becomes less important for larger resistivity.

Our calculation is valid in the $s$ - $d$ model as well, where localized magnetic moment is due to $d$ electron and the current is carried by $s$ electron.

In conclusion, we have studied the macroscopic quantum tunneling of a domain wall in a metallic ferromagnet on the basis of Hubbard model. The crucial difference from the case of an insulator is the presence of ohmic dissipation even at zero temperature due to the gapless Stoner excitation. The coupling of domain wall to electrons is effective only for momentum transfer of $|q| \lesssim \lambda^{-1}$, while Stoner excitation is gapless at the restricted region $k_{\mathrm{F} \uparrow}-k_{\mathrm{F} \downarrow}<|q|\left(<k_{\mathrm{F} \uparrow}+k_{\mathrm{F} \downarrow}\right)$. Hence the effect is negligible for a thick domain wall in which experiments so far have been carried out. On the other hand, important effects of the ohmic dissipation are expected in thinner domain walls and in weak ferromagnets, which will be within the present experimental attainability.

The authors are grateful to H. Kohno, H. Yoshioka and M. Hayashi for valuable discussions at every stage of this work. G. T. also thanks K. Nosaka for her assistance in collecting articles. This work is financially supported by Ministry-Industry Joint Research program "Mesoscopic Electronics" and by Grant-in-Aid for Scientific Research on Priority Area, "Electron Wave Interference Effects in Mesoscopic Structure" (04221101) and by Monbusho International Scientific Research Program:Joint Research "Theoretical Studies on Strongly Correlated Electron Systems" (05044037) from the Ministry of Education, Science 
and Culture of Japan. 


\section{REFERENCES}

[1] P. C. E. Stamp, E. M. Chudnovsky and B. Barbara, Int. J. Mod. Phys. B6, 1355 (1992).

[2] E. M. Chudnovsky and L. Gunther, Phys. Rev. Lett. 60, 661 (1988).

[3] E. M. Chudnovsky and L. Gunther, Phys. Rev. B37, 9455 (1988).

[4] P. C. E. Stamp, Phys. Rev. Lett. 66, 2802 (1991).

[5] D. D. Awaschalom, J. F. Smyth, G. Grinstein, D. P. DiVincenzo and D. Loss, phys. Rev. Lett. 68, 3092 (1992).

[6] A. O. Caldeira and A. J. Leggett, Phys. Rev. Lett. 46, 211 (1981); A. O. Caldeira and A. J. Leggett, Ann. Phys. 149, 374 (1983).

[7] The effect of electron was briefly touched on phenomenological ground in E. M. Chudnovsky, O. Iglesias and P. C. E. Stamp, Phys. Rev. B46, 5392 (1992).

[8] V. Korenman, J. L. Murray and R. E. Prange, Phys. Rev. B16, 4032 (1977).

[9] C. Herring, in Magnetism, edited by G. T. Rado and H. Suhl (Academic, New York,1966), Vol. IV.

[10] The results are applicable also to the case of uniaxial anisotropy if $K_{\perp}$ is replaced by the demagnetization energy $\mu_{0}(\hbar \gamma)^{2} / a^{6}$.

[11] The factor of $\epsilon^{5 / 4}$ arises from the barrier height and width in the small $\epsilon$ limit as seen by the WKB approximation; $\Gamma_{0} \propto(\text { barrier height })^{1 / 2} \times Q_{0} \propto \epsilon^{3 / 4} \epsilon^{1 / 2}$.

[12] The RPA to the interaction, $U$, is taken because we have not included in the magnetization the Coulomb interaction with finite momentum transfer $H_{\mathrm{U}}^{(\text {fast })} \equiv$ $-(U / 2) \sum_{\mathbf{q} \neq 0} \sum_{\mathbf{k k}^{\prime}}\left(a_{\mathbf{k}+\mathbf{q}}^{\dagger} \sigma_{z} a_{\mathbf{k}}\right)\left(a_{\mathbf{k}^{\prime}-\mathbf{q}}^{\dagger} \sigma_{z} a_{\mathbf{k}^{\prime}}\right)$.

[13] K. Awaka, G. Tatara and H. Fukuyama, Jour. Phys. Soc. Jpn. 62, 1939 (1993).

[14] This cutoff is needed since excitated states of variable $Q$ around the metastable mini- 
mum is not taken into account in the instanton calculation. See Yu. Kagan and N. V. Prokof'ev, Sov. Phys. JETP 63, 1276 (1986).

[15] C. Paulsen, L. C. Sampaio, B. Barbara, D. Fruchard, A. Marchand, J. L. Tholence and M. Uehara, Phys. Lett. A161, 319 (1991); C. Paulsen, L. C. Sampaio, B. Barbara, R. T-Tachoueres, D. Fruchart, A. Marchand, J. L. Tholence and M. Uehara, Europhys. Lett. 19, 643 (1992).

[16] M. Uehara, B. Barbara, B. Dieny and P. C. E. Stamp, Phys. Lett. A114, 23 (1986); M. Uehara and B. Barbara, J. Physique, 47,235 (1986). 


\section{FIGURES}

FIG. 1. Configuration of a planar domain wall.

FIG. 2. Strength of ohmic dissipation $\eta$ given by Eq. (9) as a function of the width of the wall $\lambda / a, a$ being a lattice constant, for three choices of $\delta \equiv\left(k_{\mathrm{F} \uparrow}-k_{\mathrm{F} \downarrow}\right) /\left(k_{\mathrm{F} \uparrow}+k_{\mathrm{F} \downarrow}\right)=0.05,0.1$ and 0.2 with $\left(k_{\mathrm{F} \uparrow}+k_{\mathrm{F} \downarrow}\right) a=6.0$.

FIG. 3. The tunneling rate $\Gamma$ for the insulating $(\eta=0)$ (dashed line) and the metallic $(\eta=0.1)$ (solid line) magnet as a function of $\epsilon, H_{c}$ being the coercive field. Number of spin is $N=10^{4}$ and $10^{8}$. 